\title{
Investigation of Physical Properties of Graphene-Cement Composite for Structural Applications
}

\author{
Ahmadreza Sedaghat ${ }^{1 *}$, Manoj K. Ram ${ }^{2}$, A. Zayed ${ }^{1}$, Rajeev Kamal ${ }^{3}$, Natallia Shanahan ${ }^{1}$ \\ ${ }^{1}$ Department of Civil and Environmental Engineering, University of South Florida, Tampa, USA; ${ }^{2}$ Nanotechnology Research and \\ Education Center, Clean Energy Research Center, University of South Florida, Tampa, USA; ${ }^{3}$ Department of Chemical \& Biomedi- \\ cal Engineering, University of South Florida, Tampa, USA. \\ Email: asedagha@mail.usf.edu, mkram@usf.edu, zayed@usf.edu, rajeev@mail.usf.edu, nkashalo@mail.usf.edu
}

Received October $14^{\text {th }}, 2013$; revised November $14^{\text {th }}, 2013$; accepted November $20^{\text {th }}, 2013$

Copyright (C) 2014 Ahmadreza Sedaghat et al. This is an open access article distributed under the Creative Commons Attribution License, which permits unrestricted use, distribution, and reproduction in any medium, provided the original work is properly cited. In accordance of the Creative Commons Attribution License all Copyrights (C) 2014 are reserved for SCIRP and the owner of the intellectual property Ahmadreza Sedaghat et al. All Copyright (C) 2014 are guarded by law and by SCIRP as a guardian.

\section{ABSTRACT}

The hydration of cement generates heat due to the exothermic nature of the hydration process. Poor heat dissipation in mass concrete results in a temperature gradient between the inner core and the outer surface of the element. High temperature gradients generate tensile stresses that may exceed the tensile strength of concrete thus leading to thermal cracking. The present paper is an attempt to understand the thermal (heat sink property) and microstructural changes in the hydrated graphene-Portland cement composites. Thermal diffusivity and electrical conductivity of the hydrated graphene-cement composite were measured at various graphene to cement ratios. The mass-volume method was implemented to measure the density of the hydrated graphene-cement composite. Particle size distribution of Portland cement was measured by using a laser scattering particle size analyzer. Heat of hydration of Portland cement was assessed by using a TAMAIR isothermal conduction calorimeter. Scanning electron microscopy (SEM) was implemented to study microstructural changes of the hydrated graphene-cement composites. The mineralogy of graphene-cement and the hydrated graphene-cement composites was investigated by using $\mathrm{X}$-ray diffraction. The findings indicate that incorporation of graphene enhances the thermal properties of the hydrated cement indicating a potential for reduction in early age thermal cracking and durability improvement of the concrete structures.

\section{KEYWORDS}

Ceramics; Composite Materials; Graphene; Electrical Conductivity; Thermal Conductivity

\section{Introduction}

Concrete is a composite material of aggregates and binders where binding materials are primarily a combination of Portland cement, pozzolanic materials and water [1,2]. Hydration of cement generates heat due to the exothermic nature of the hydration process. The phases mainly responsible for heat generation during the hydration process are tricalcium silicate $\left(\mathrm{C}_{3} \mathrm{~S}\right)$, dicalcium silicate $\left(\mathrm{C}_{2} \mathrm{~S}\right)$, tricalcium aluminate $\left(\mathrm{C}_{3} \mathrm{~A}\right)$ and tetracalcium aluminoferrite $\left(\mathrm{C}_{4} \mathrm{AF}\right)$ [2,3]. The hydration process of Portland cement depends on several factors or parameters such as cement mineralogical composition, particle size distribu-

\footnotetext{
"Corresponding author.
}

tion, water to cement ratio and curing temperature. Due to the exothermic nature of the reaction combined with poor heat dissipation in massive concrete elements, the hydration process results in a temperature gradient between the inner core and the outer surface of the element [4]. The high temperature gradient is known to result in large tensile stresses that may exceed the tensile strength of concrete thus leading to thermal cracking. The temperature gradient minimization in an element could be achieved through lowering the temperature rise due to hydration and/or improving heat dissipation by increasing thermal conductivity of concrete. Improving the paste thermal conductivity reduces the temperature gradient in the concrete element, thus reducing the probability of 
concrete thermal cracking [5].

Recent research indicates the possibility of using nanomaterials (carbon nanotube, graphene, titanium oxide, nanosilica, and nanoalumina) in civil infrastructure applications; however, costly process and low production of such materials may limit such applications [6]. Incorporation of nanomaterials changes the macroscopic properties of the main binder, namely, Portland cement paste [7]. Introduction of nanomaterials in cement paste reduces the porosity and rate of hydration leading to the development of stronger and more durable products [7]. The structure of the hydrated gel is also affected by the introduction of nanomaterials at a nano-level $[8,9]$. The long term creep properties of cement paste are dependent on the density of calcium silicate hydrate which is the main hydration product. Introduction of nanomaterials in concrete using an electromutagenic process modifies the microstructure of high performance concrete without changing the dimensions or appearance [10]. High surface area of the nanomaterials makes them efficient in controlling the propagation of microcracks in cementitious composite materials. Defects present in the lattice structure of the carbon nanotubes, provide potential sites for formation of carboxyl (-COOH) and hydroxyl (-OH) species and creation of bonding to the hydrated cement [11]. It is demonstrated that graphene-oxide (GO) nanosheets may reduce the brittleness and enhance toughness, tensile and flexural strength of the hydrated cement composite. GO can regulate cement hydration and distinctly affect the mechanical properties of hydrated cement composite [12].

In addition to increasing strength, preventing cracking and reducing porosity, nanomaterials are useful as anticorrosive agents in reinforced concrete. Recently, it has been shown that titanium addition to cementitious binders results in triggering self-cleaning process in cement pastes [13]. Carbon nanotubes, nanoflakes or carbon block additions were used in electromagnetic shielding applications [14]. Carbon nanotubes, with extremely high aspect ratios (length to diameter ratio), are distributed in a much finer scale relative to other common fibers resulting in efficient bridging in hydrated cement composite and reduction of microcrack propagation [15]. The functionalized carbon nanotubes (F-CNT), showing hydrophilic behavior, can interfere with the cement hydration mechanism and may improve or reduce the performance of hydrated cement. The extent of this process is dependent upon the amount of F-CNT incorporated into the composite mix [16].

Nanomaterials such as nanoalumina are found to improve the flexural strength of concrete $[17,18]$. Titanium and nanosilica enhance abrasion resistance and flexural strength $[19,20]$. Nanosilica has been effective in promoting early precipitation of calcium silicate hydrate thus shortening the induction period [21,22]. Incorporation of nanomaterials affects the cement hydration process and the rate of formation of hydration products enhancing the quality performance of concrete.

Graphene, a 2-D $\pi$-conjugation, has several extraordinary physical properties such as high thermal conductivity, high electrical conductivity, high surface area (2630 $\mathrm{m}^{2} / \mathrm{g}$ ), high elastic modulus and ampi-polar electric field effect [23-25]. Graphene forms a colloidal mixture and has also been used in making nanocomposites with conducting polymer for supercapacitor applications [26-28]. In the current study, graphene was introduced as a partial replacement of Portland cement at various ratios to understand its effect on the heat dissipation in cementitious paste during the cement hydration. Thermal diffusivity and electrical conductivity of the hydrated cement paste incorporating different quantities of graphene were measured to understand thermal and electrical properties of the composite. SEM and X-ray diffraction methods were used to understand the physical and structural properties of the graphene-cement composite.

\section{Experimental}

\subsection{As-Received Materials}

A commercially available Portland cement and graphene platelets of $110 \times 110 \times 0.12 \mathrm{~nm}$ (Angstrom Materials, N008-100-N) were used in this study. All other chemicals and materials were used as purchased without any modifications.

\subsection{Composite Materials Preparation}

Hydrated graphene-cement mixes were prepared using a commercial mixer (Speedmixer DAC 150.1 FVZ) with constant water to solid ratio of 0.5 and at ambient temperature of $23^{\circ} \mathrm{C} \pm 2^{\circ} \mathrm{C}$. Sufficient workability of the mix could be obtained at a water to solid ratio of 0.5 . The composite was mixed for 3 minutes in the Speedmixer operated at 3500 RPM. The mixes were poured into small containers and wrapped with plastic tape to avoid evaporation of water and desiccation. The mixes were cured for 44 hours from the mixing time. This hydration time was selected as it corresponds to the approximate average time at which the concrete element experiences a large temperature gradient between its inner core and outer surface [5].

\subsection{Materials Characterization}

The main constituent responsible for temperature rise in a concrete element is Portland cement due to the exothermic nature of its reaction with water. In defining temperature rise in mass elements, equally important to the ability to dissipate the heat is the amount of heat gener- 
ated by Portland cement hydration. Cement fineness and mineralogy are the main contributors to the total heat generated through the cement hydration process. In conducting the current research, it is therefore important to characterize the as-received cement properties that are of significance to temperature rise; namely, cement fineness and mineralogy. Mineralogical composition of Portland cement was studied using X-ray diffraction. The diffractometer used in this study was a PANalytical Cubix Pro coupled with HighScore Plus software for crystalline phase analysis. The software uses Rietveld analysis for phase quantification. The tube was operated at a current of $40 \mathrm{~mA}$ and a voltage of $45 \mathrm{KV}$. The scan range was set for $2 \theta$ of $8^{\circ}-70^{\circ}$ using a step size of $0.014^{\circ}$ with the time per step of 10 seconds. For hydrated composites and powdered specimens, rutile was added as an internal standard at $10 \%$ by weight of the sample for qualitative comparison. Additionally, heat of hydration measurements on portland cement was conducted using TAMAIR isothermal conduction calorimeter instrument with 8-twin channels at a bath temperature of $23^{\circ} \mathrm{C}$. The test was conducted in accordance with the internal mixing procedure as outlined in the ASTM C1702 [29]. A Horiba LA-950 laser scattering particle size analyzer was used to assess the particle size distribution of the as- received cement.

The microstructure of the hydrated graphene-cement composites was examined using Hitachi SU-70 scanning electron microscope. For electrical conductivity measurements, cylindrical pellets of ground hydrated graphenecement composites were prepared with a constant mass of 0.63 grams, a circular diameter of $13.07 \mathrm{~mm}$ and a thickness of $2.6 \pm 0.1 \mathrm{~mm}$. The pellets were oven dried at $105^{\circ} \mathrm{C}$ to eliminate the contribution of evaporable water to electrical conductivity. The pellets were gradually loaded up to 10 kips in a period of 3 minutes then unloaded for another 3 minutes before taking measurements. The electrical conductivity of the pellets was measured by setting them between two metal plates. The current was measured at different voltages using a Keithley electrometer 2400 . The conductivity was calculated based on the current, voltage and the dimensions of the pellet samples.

In examining the effectiveness of graphene to improve concrete heat dissipation, thermal diffusivity was also measured. Hydrated graphene-cement composite specimens with thickness of $1.5 \pm 0.05 \mathrm{~mm}$ and diameter of $10 \pm 0.1 \mathrm{~mm}$ were prepared and cured for 44 hours. Thermal diffusivity was determined using Linseis (c) XFA500 instrument conforming to ASTM E-1461, DIN 30905 and DIN EN 821 specifications. The instrument provides results with $\pm 5 \%$ accuracy for most homogenous materials tested based on the flash method procedure [30].

Figure 1 reveals the morphology of hydrated graphene-Portland cement and possible nanocomposite structure using SEM technique. The emphasis is given to how the graphene is attached to the main portland cement hydration products such as calcium silicate hydrate and calcium hydroxide.

\section{Results and Discussion}

\subsection{Cement Characterization}

The X-ray diffraction pattern of the as-received cement is presented in Figure 2. Rietveld analysis indicates that the amounts of the main crystalline phases are: alite $=59.7 \%$, belite $=12.6 \%$, tricalcium aluminate $=11.2 \%$, tetracalcium alumino-ferrite $=6.0 \%$, gypsum $=3.6 \%$, calcite $=$ $2.3 \%$, periclase $=1.7 \%$ and arkanite $=1.6 \%$. The amount of tricalcium aluminate $\left(\mathrm{C}_{3} \mathrm{~A}\right)$ present in this cement is high and it is therefore expected that this cement would generate higher heat of hydration compared to moderateheat cements. ASTM C-150 [31] sets a maximum of $8 \%$ on $\mathrm{C}_{3} \mathrm{~A}$ for moderate-heat Portland cements. It is therefore anticipated that a concrete element incorporating this cement could generate a higher temperature gradient unless there is significant improvement in heat dissipation to counteract the effect of higher heat generation.

The particle size distribution analysis presented in Figure 3 indicates that the cement has a mean, median, mode and standard deviation of $10.27 \mu \mathrm{m}, 9.08 \mu \mathrm{m}, 10.82$ $\mu \mathrm{m}$ and $6.97 \mu \mathrm{m}$ respectively, with $70.5 \%$ of the as-received cement particles laying within one standard deviation of the reported mean. The reported value of the mean is the equivalent spherical diameter of the cement particles measured on volume basis. It is noteworthy that advanced Horiba hardware and software do not require normal or Rosin-Rammler [32] distribution assumptions to establish the particle size distribution curve.

Figure 4 shows the total heat generated at seven days of hydration for Portland cement paste to be $406 \mathrm{~J} / \mathrm{g}$. The data indicate that more than $75 \%$ of the seven-day heat of hydration is generated during the first 48 hours. This implies that the potential for temperature rise is more important during the first few days. It also indicates the significance of increasing thermal diffusivity during the first few days to reduce the cracking potential of a massive concrete element.

\subsection{X-Ray Diffraction and Rietveld Analysis}

X-ray diffraction patterns of cement and graphene-cement composites are presented in Figure 5 for hydrated and anhydrous specimens. Rutile was added to the specimens as an internal standard at a $10 \%$ by weight of the solids. Figure 5(a) shows the XRD patterns of anhydrous graphene-cement composites and cement powder. The main diffraction peak for graphene occurs at a diffraction angle of $26.56^{\circ}$. The intensity ratio of graphene to titanium oxide $\left(2 \theta=27.45^{\circ}\right)$ increases with the increase of 


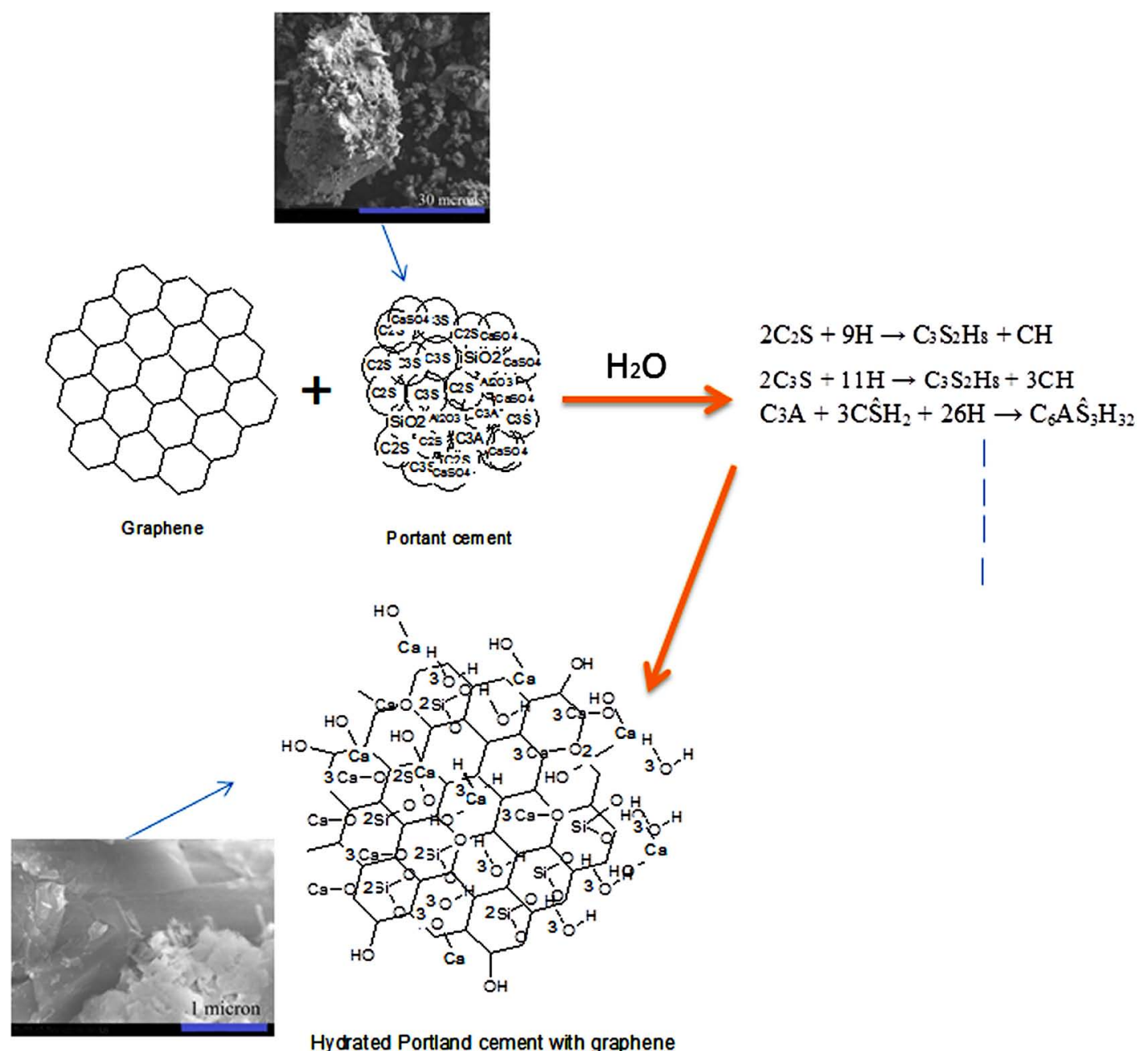

Figure 1. Schematic of hydrated graphene-cement composite and possible nanocomposite structure.

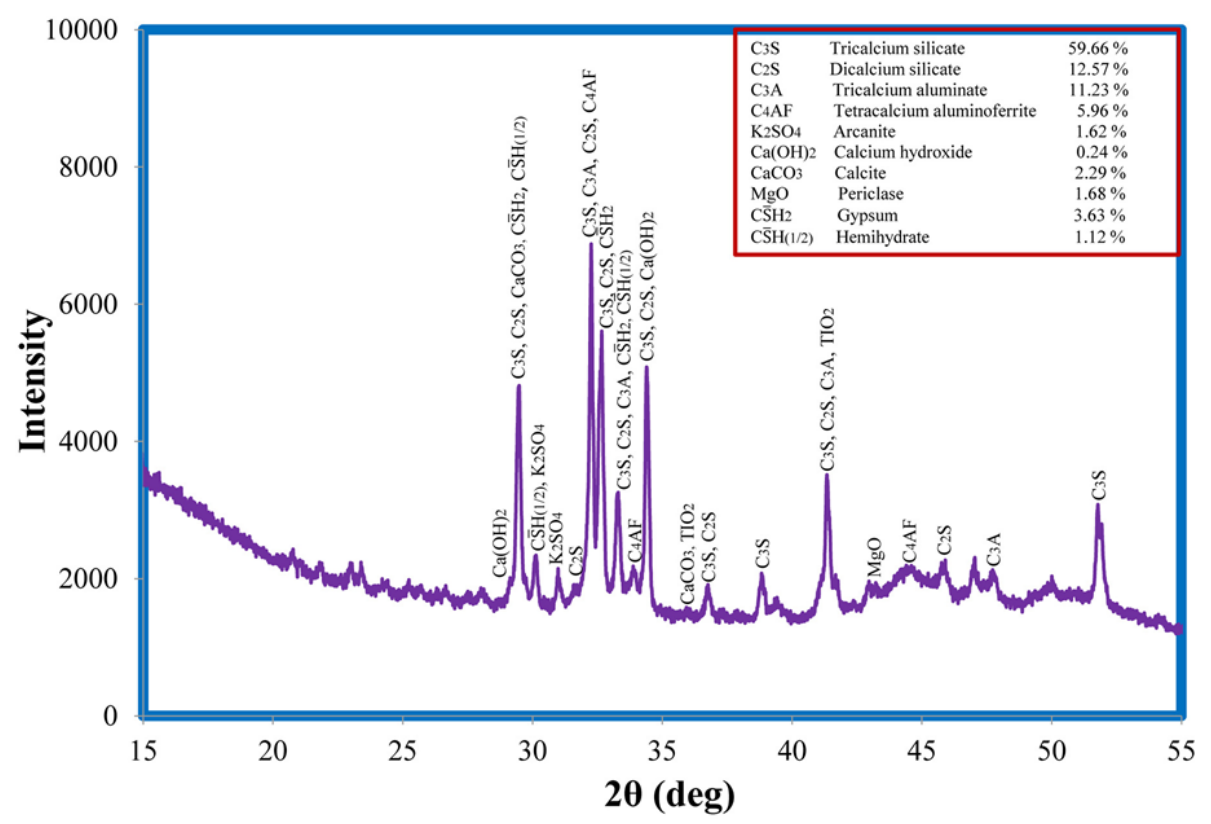

Figure 2. Mineralogical analysis of as-received cement using XRD. 


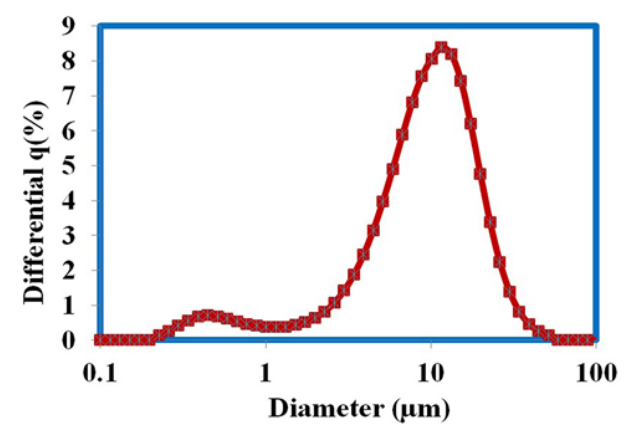

Figure 3. Particle size distribution of as-received cement.
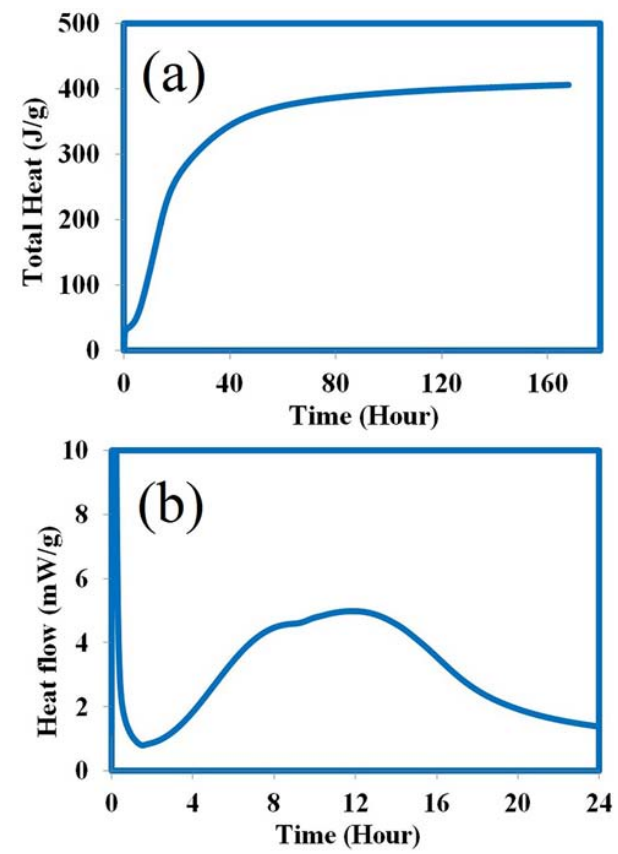

Figure 4. Cement paste (a) total heat \& (b) heat flow.

graphene content in the composite specimen. A hump can be observed at $2 \theta$ of $42^{\circ}-52^{\circ}$ and is more prominent at $10 \%$ graphene content while absent in the cement powder specimen with no graphene. Figure 5(b) for hydrated samples shows fewer and shorter peaks between $15^{\circ}$ to $30^{\circ}$ due to the chemical reaction of water with cement phases and formation of poorly crystalline calcium silicate hydrate gel in addition to other hydration phases such as calcium hydroxide and ettringite [1]. The characteristic peak of $\mathrm{Ca}(\mathrm{OH})_{2}$ at $18^{\circ}$ is also clearly visible. The presence of a sharp peak of graphene from (002) plane, due to incremental increase of graphene in hydrated graphene-cement composite, is clearly shown at $2 \theta=26.56^{\circ}$.

\subsection{Temperature Treatment of Hydrated Graphene-Cement Composites}

The effect of temperature on the hydrated graphene-ce- ment samples is shown in Figure 6. Mixes of different ratios of graphene to cement, hydrated for 44 hours, were treated at varying temperatures of $23^{\circ} \mathrm{C}, 100^{\circ} \mathrm{C}, 400^{\circ} \mathrm{C}$, and $600^{\circ} \mathrm{C}$ to $750^{\circ} \mathrm{C}$. The presence of graphene in composite was studied by capturing the images of the mix at different temperatures. Figures 6(A)-(D) shows the composites containing $0 \%, 1 \%, 5 \%, 10 \%$ graphene at $23^{\circ} \mathrm{C}$. Figures 6(E)-(H) shows no apparent difference in composites containing $0 \%, 1 \%, 5 \%, 10 \%$ graphene heated at $100^{\circ} \mathrm{C}$. The varying color intensity in the pictures at different ratios of graphene is due to the incremental increase of carbon material in the composites. Presence of water in the graphene-cement mixes at $23^{\circ} \mathrm{C}$ is reflected in the images as extra transparency compared to other mixes heated at higher temperatures. Figures 6(I)-(L) shows the images of same composition of graphene-cement heated at $400^{\circ} \mathrm{C}$. The smooth structure observed in the images is probably due to the evaporation of capillary pore water and decomposition of calcium silicate hydrate in the mixes. The mixes heated beyond $400^{\circ} \mathrm{C}$ are shown in Figures 6(M)-(T). Interestingly, the graphene has been found to oxidize when heated to $600^{\circ} \mathrm{C}$ and $750^{\circ} \mathrm{C}$. Also, calcium hydroxide decomposes in the temperature range of $400^{\circ} \mathrm{C}-500^{\circ} \mathrm{C}$. It appears that the elemental metallic oxides in cement act as catalysts contributing to graphene oxidation regardless of the percentage of graphene present in the cement based composites. The hydration process may cause temperature gradient of $30^{\circ} \mathrm{C}$ to $90^{\circ} \mathrm{C}$ in massive concrete elements [5]. In this study, the hydrated graphene cement composite was examined at higher temperatures to investigate the physical changes that may occur in the composite in the event that the concrete element is exposed to external high temperatures (including fire).

\subsection{Morphological Properties of Composite Materials in Hydration}

The SEM image of the hydrated cement is shown in Figures 7(A)-(C). The structure of the hydrated cement shows the formation of the needle-like ettringite and the sheet-like habit of calcium hydroxide $\left(\mathrm{Ca}(\mathrm{OH})_{2}\right)$. Figures 7(D)-(F) shows a mix of $1 \%$ graphene and $99 \%$ cement in the hydrated form. The structure of the 1\% graphene and 99\% cement mix shown in Figures 7(D)(F) is found to be different from the hydrated cement samples. Figures 7(G)-(I) showing the hydrated sample of $5 \%$ graphene and $95 \%$ cement mix is more compact, with less needle-like formations, grown in the hydrated samples. The increase of graphene may decrease the porosity of the hydrated product as the graphene nanoparticles fill the micro-size capillary pores. It is also possible that graphene has an effect on the morphology of the needle-shaped ettringite. Figures 7(J)-(L) depicts images of the hydrated $10 \%$ graphene and $90 \%$ cement mix which 

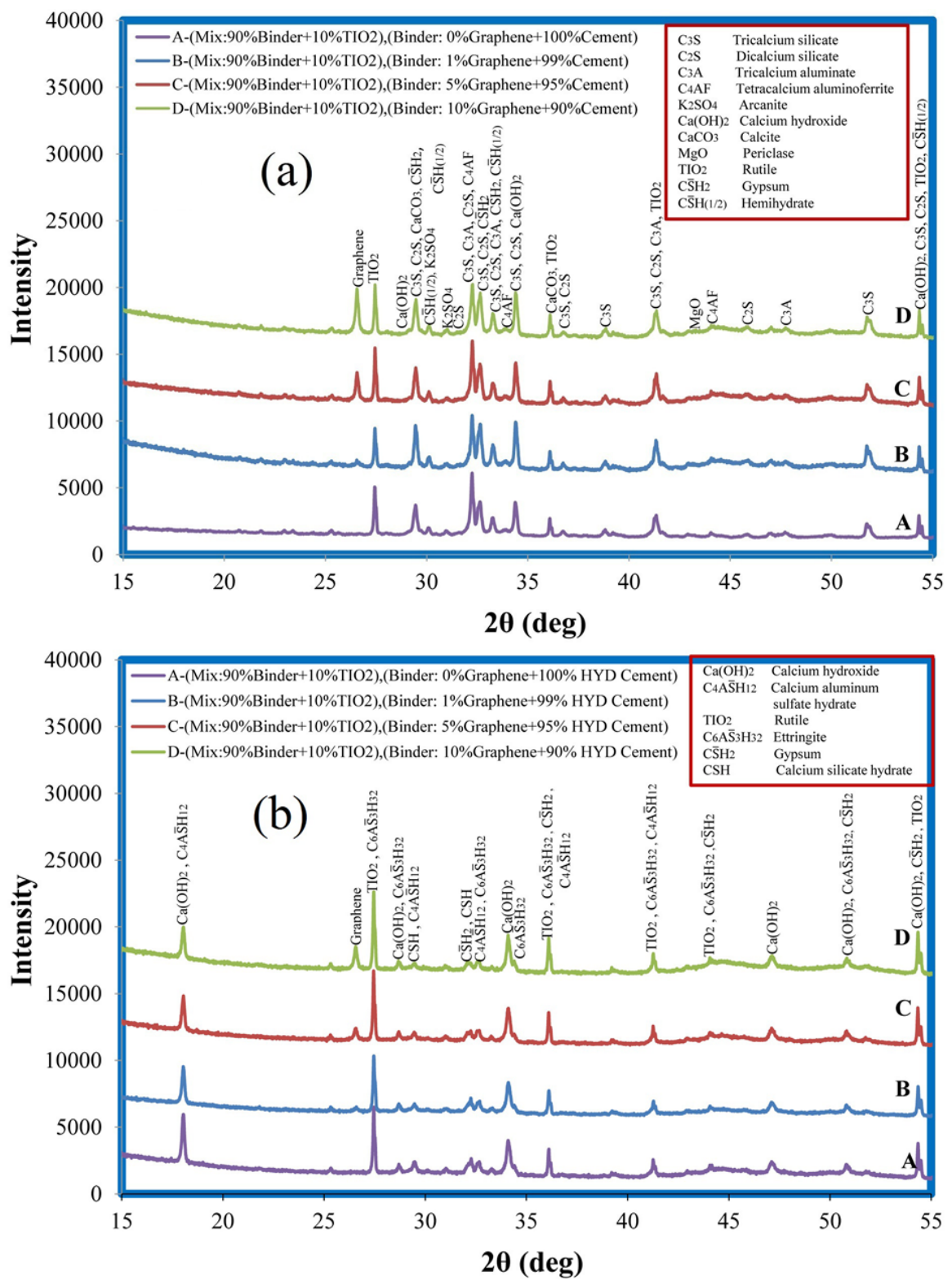

Figure 5. XRD patterns of cement \& graphene-cement composites (a) anhydrous \& (b) hydrated.

reveal no growth of needle-shaped structure, while the compact structure is predominant. Drastic reduction of porosity is anticipated for such a composite.

\subsection{Electrical Conductivity Properties of Composite Materials in Hydration}

Figure 8 and Table 1 show the effect of graphene content on the electrical resistivity of the hydrated graphenecement samples. The conductivity of hydrated cement paste was approximately $10^{-8} \mathrm{~S} / \mathrm{m}$; however, incorporation of $1 \%$ of graphene changes the conductivity by 3 orders of magnitude. Interestingly, the increase in conductivity is substantial when the composite contains $5 \%$ graphene. At a graphene content of $10 \%$, the conductivi- ty measured was at about $10^{-2} \mathrm{~S} / \mathrm{m}$. The increase in conductivity with graphene content appears to be accompanied by a change in electrical properties from insulating to semiconducting behavior. Such an increase in conductivity could bring about wide range of electrical applications for graphene-cement composites. The results indicate that low additions of graphene, even at $1 \%$, could be sufficient for use in applications where electrostatic dissipation (ESD) is desirable.

\subsection{Thermal Diffusivity Properties of Composite Materials in Hydration}

Thermal diffusivity for the composite samples was determined using Parker’s formula [30]: 


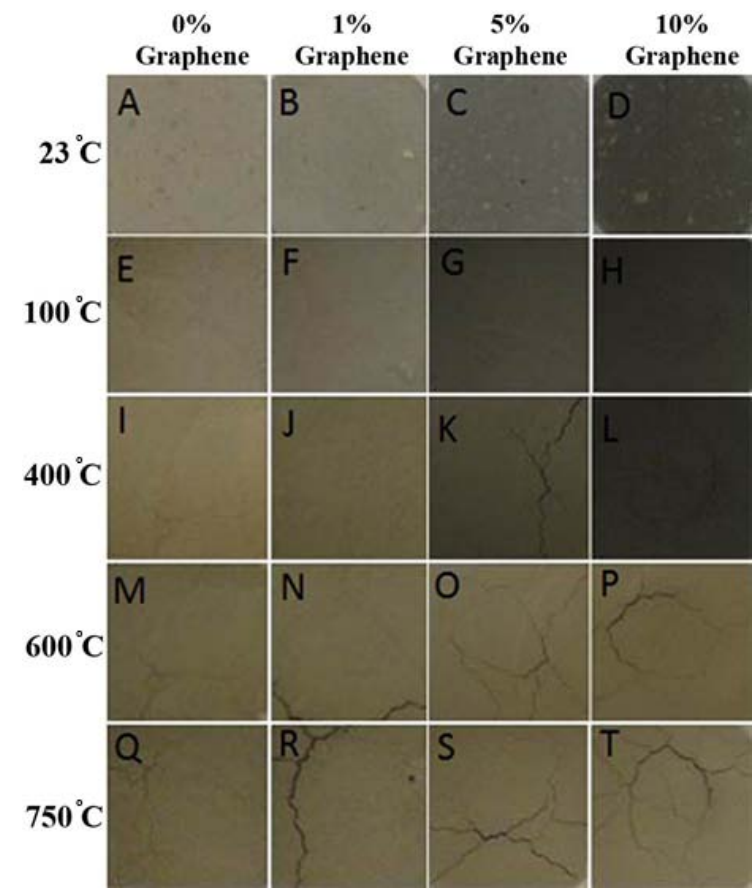

Figure 6. Temperature treatment of hydrated graphene-cement composites.

$$
\alpha=\frac{0.139 d^{2}}{t_{\left(\frac{1}{2}\right)}}
$$

Where

$(\alpha)=$ thermal diffusivity in $\left(\mathrm{m}^{2} / \mathrm{s}\right)$;

$t_{(1 / 2)}=$ time (s) to reach $50 \%$ of maximum temperature amplitude;

$d=$ thickness of the material (m) across the direction of heat flow.

About three runs were taken at every temperature for a better estimation of the thermal diffusivity for the composite samples. The hydrated graphene-cement composites were tested under a similar range of temperatures as shown in Figure 9. The general trend observed here is that there is a decrease in thermal diffusivity with an increase in temperature, from $25^{\circ} \mathrm{C}$ to $400^{\circ} \mathrm{C}$. It appears that the decrease in thermal diffusivity is about 35\% for all the mixes, regardless of the graphene content. The data indicate that incorporation of $1 \%$ graphene did not have any significant effect on thermal diffusivity of the mix. Incorporation of $5 \%$ graphene, on the other hand, improved the thermal diffusivity by $25 \%$ at $25^{\circ} \mathrm{C}$ and about $30 \%$ at $400^{\circ} \mathrm{C}$ compared to the pure cement paste

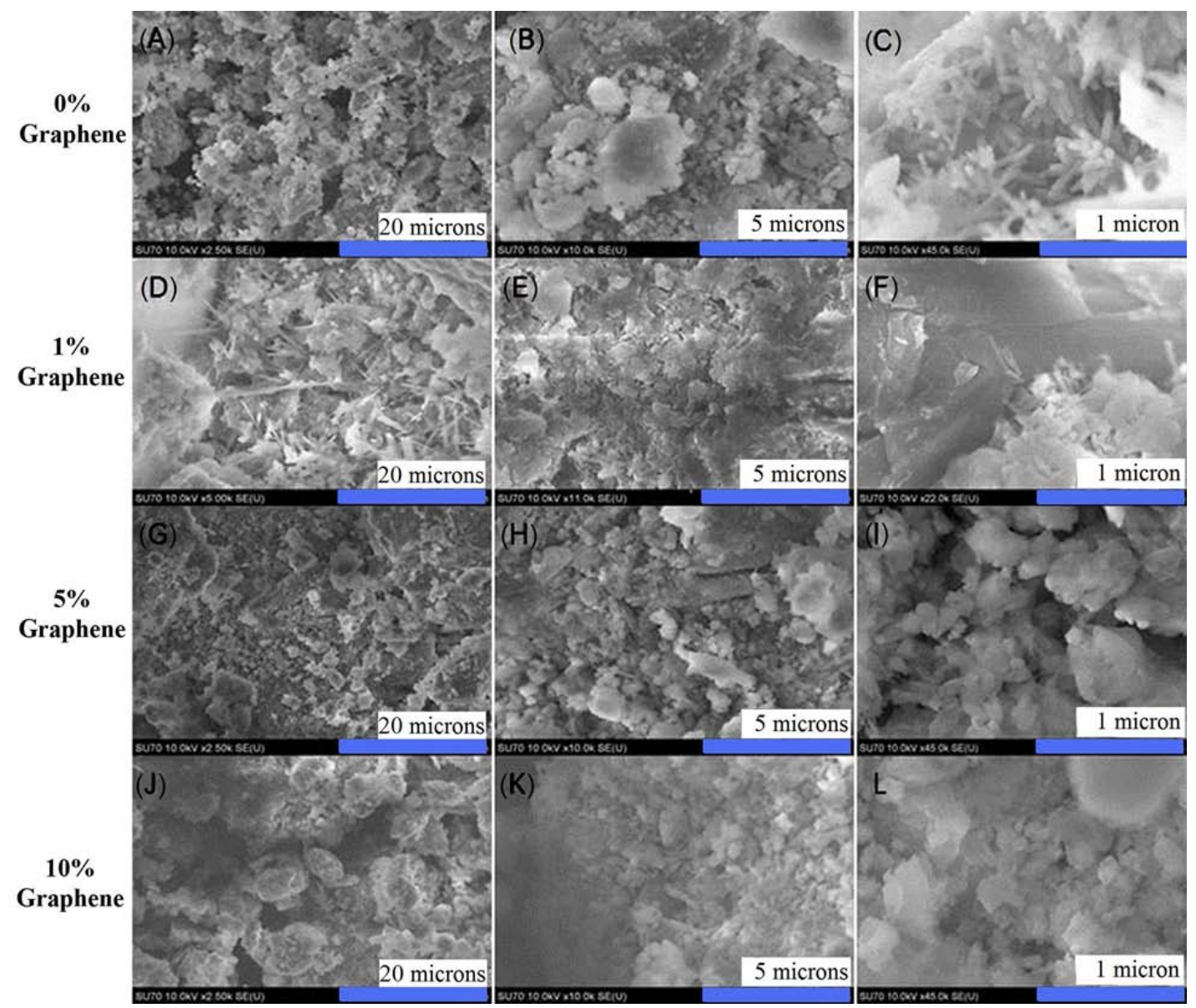

Figure 7. Scanning electron microscopy image of hydrated graphene-cement composite. 


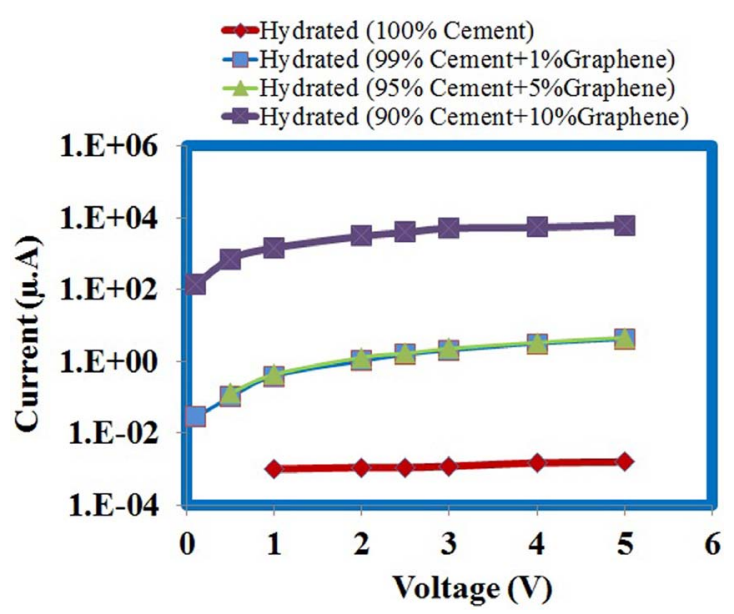

Figure 8. Electrical conductivity of hydrated graphene-cement composites. or the $1 \%$ graphene composite. The mix containing $10 \%$ graphene shows significant improvement in thermal diffusivity of about $75 \%$ at $25^{\circ} \mathrm{C}$ and $60 \%$ at $400^{\circ} \mathrm{C}$. In general, it appears that incorporation of graphene in cement paste could significantly improve thermal diffusivity of the composite. Improvement of thermal diffusivity of cementitious pastes can reduce the temperature gradient $\left(30^{\circ} \mathrm{C}-90^{\circ} \mathrm{C}\right)$ effect due to cement hydration in mass concrete structures. This can consequently reduce the potential of massive concrete elements to experience thermal cracking thus improving thermal integrity and durability of concrete structures.

\section{Conclusion}

Incorporation of graphene nanoparticles in cement paste showed interesting modifications in microstructural,

Table 1. Hydrated graphene-cement composite properties.

\begin{tabular}{|c|c|c|c|c|}
\hline Mix characteristics & $\begin{array}{l}\text { Resistivity } \\
(\Omega \cdot \mathrm{m})\end{array}$ & $\begin{array}{l}\text { Conductivity } \\
\left(\mathrm{S} \cdot \mathrm{m}^{-1}\right)\end{array}$ & $\begin{array}{l}\text { Oven dried bulk } \\
\text { density }\left(\mathrm{g} / \mathrm{cm}^{3}\right)\end{array}$ & $\begin{array}{l}\text { Encapsulated bulk densi- } \\
\text { ty }\left(\mathrm{g} / \mathrm{cm}^{3}\right)\end{array}$ \\
\hline Hydrated (100\% Cement) & $112,441,765$ & $8.89 \mathrm{E}-09$ & 1.490 & 1.851 \\
\hline Hydrated (99\% Cement + 1\% Graphene) & 121,820 & $8.21 \mathrm{E}-06$ & 1.481 & 1.847 \\
\hline Hydrated (95\% Cement + 5\% Graphene) & 94,659 & $1.06 \mathrm{E}-05$ & 1.463 & 1.838 \\
\hline Hydrated (90\% Cement + 10\% Graphene) & 37 & $2.70 \mathrm{E}-02$ & 1.436 & 1.827 \\
\hline
\end{tabular}

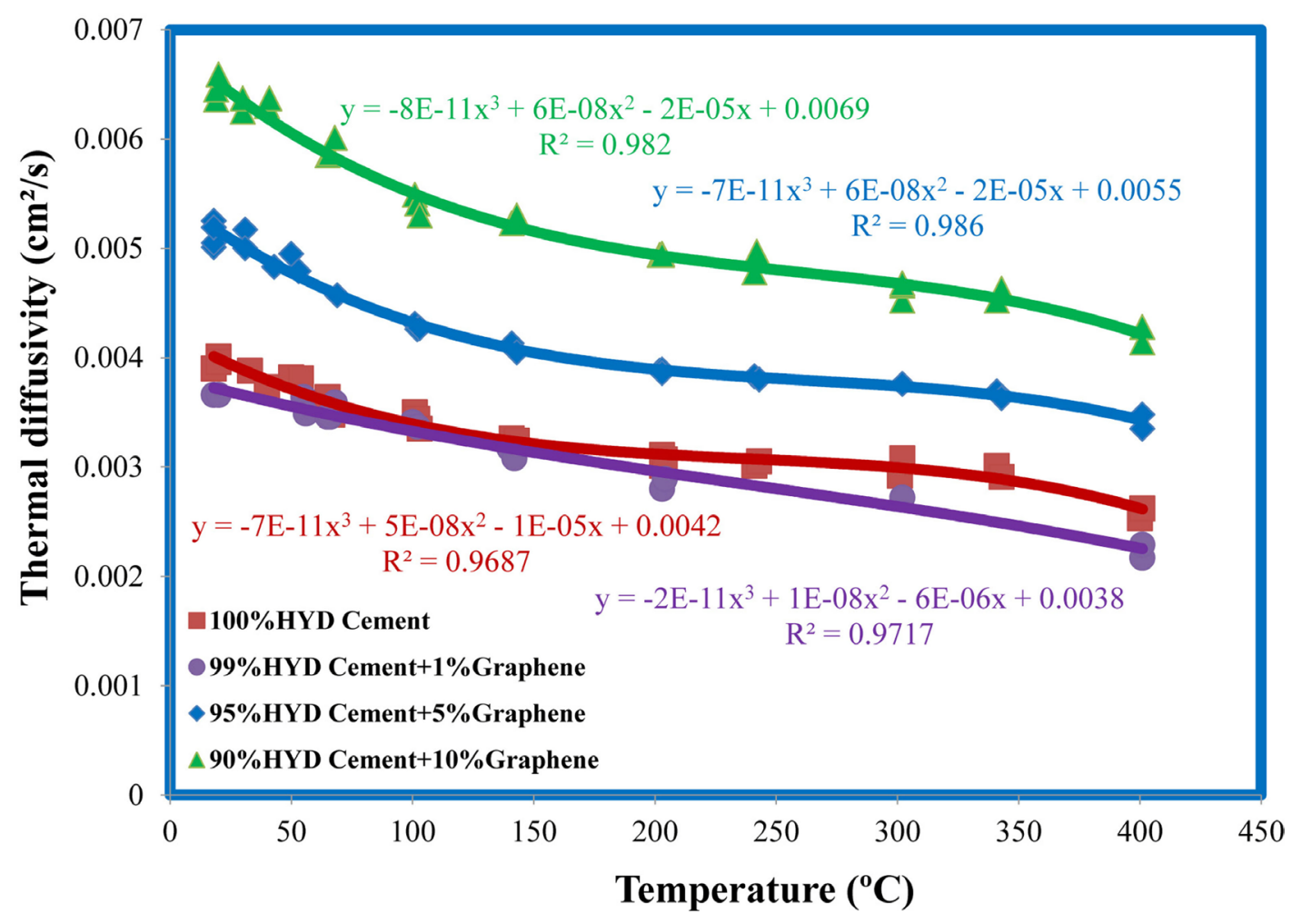

Figure 9. Thermal diffusivity of hydrated graphene-cement composites. 
morphological, electrical and thermal properties of the paste. Thermal diffusivity and electrical conductivity were found to increase with increasing the graphene content in the composite. The increase in thermal diffusivity of the hydrated graphene cement composite is a clear indication of the heat sink capacity of graphene. This effect is of significant importance especially during the exothermic reactions taking place during the initial stages of hydration of portland cement. The hydrated graphene-cement samples indicate the presence of graphitic plane in the composite structure. The rod or needle-shaped morphology of ettringite, which is typically observed in hydrated cement paste, was less prevalent in the graphene composites and appeared to be affected by graphene content. The metal oxides in cement act as a catalyst for the oxidation of graphene at higher temperatures $\left(600^{\circ} \mathrm{C}\right.$ to $750^{\circ} \mathrm{C}$ ), regardless of the quantity of graphene present in cement-based composite. The impact of the incremental increase of graphene on the electrical conductivity of the composites indicates the potential of using graphene in application where electrostatic dissipation (ESD) of charge is desirable.

\section{REFERENCES}

[1] S. Mindess, J. F. Young and D. Darwin, “Concrete,” 2nd Edition, Prentice-Hall, Upper Saddle River, 2003.

[2] I. Odler, "Lea's Chemistry of Cement and Concrete," 4th Edition, Arnold Publishers, London, 1998, pp. 241-297.

[3] A. Sedaghat, A. Zayed and P. Sandberg, "Measurement and Prediction of Heat of Hydration of Portland Cement Using Isothermal Conduction Calorimetry," Journal of Testing and Evaluation, Vol. 41, No. 6, 2013, 8 p. http://dx.doi.org/10.1520/JTE20120272

[4] M. Azenha and R. Faria, "Temperatures and Stresses due to Cement Hydration on the R/C Foundation of a Wind Tower-A Case Study,” Engineering Structures, Vol. 30, No. 9, 2008, pp. 2392-2400. http://dx.doi.org/10.1016/j.engstruct.2008.01.018

[5] A. K. Schindler, "Concrete Hydration, Temperature Development, and Setting at Early-Ages," Ph.D. Dissertation, University of Texas at Austin, Austin, 2002.

[6] H. Alkhateb, A. Al-Ostaz, A.-D. Cheng and X. Li, "Materials Genome for Graphene-Cement Nanocomposites," Journal of Nanomechanics and Micromechanics, Vol. 3, No. 3, 2013, pp. 67-77. http://dx.doi.org/10.1061/(ASCE)NM.2153-5477.000005 $\underline{5}$

[7] J. Makar, J. Margeson and J. Luh, "In Carbon Nanotube/ Cement Composites-Early Results and Potential Applications," Proceedings of the 3rd International Conference on Construction Materials: Performance, Innovations and Structural Implications, Vancouver, 22-24 August 2005, pp. 1-10.

[8] M. Vandamme and F. J. Ulm, "Nanogranular Origin of Concrete Creep,” Proceedings of the National Academy of Sciences, Vol. 106, No. 26, 2009, pp. 10552-10557. http://dx.doi.org/10.1073/pnas.0901033106

[9] F. Ulm, "Green Concrete: Nanoengineered Materials Could Reduce Greenhouse-Gas Emissions,” Technology Review, Vol. 110, No. 4, 2007, pp. 27-29.

[10] H. E. Cardenas, "Nanomaterials in Concrete: Advances in Protection, Repair, and Upgrade,” DEStech Publications, Inc., Lancaster, 2012.

[11] A. Peyvandi, P. Soroushian, N. Abdol and A. M. Bala Chandra, "Surface-Modified Graphite Nanomaterials for Improved Reinforcement Efficiency in Cementitious Paste," Carbon, Vol. 63, 2013, pp. 175-186. http://dx.doi.org/10.1016/j.carbon.2013.06.069

[12] S. Lv, Y. Ma, C. Qiu, T. Sun, J. Liu and Q. Zhou, "Effect of Graphene Oxide Nanosheets of Microstructure and Mechanical Properties of Cement Composites," Construction and Building Materials, Vol. 49, 2013, pp. 121127. http://dx.doi.org/10.1016/j.conbuildmat.2013.08.022

[13] M. V. Diamanti, M. Ormellese and M. P. Pedeferri, "Characterization of Photocatalytic and Superhydrophilic Properties of Mortars Containing Titanium Dioxide," Cement and Concrete Research, Vol. 38, No. 11, 2008, pp. 1349-1353. http://dx.doi.org/10.1016/j.cemconres.2008.07.003

[14] D. Chung, “Comparison of Submicron-Diameter Carbon Filaments and Conventional Carbon Fibers as Fillers in Composite Materials,” Carbon, Vol. 39, No. 8, 2001, pp. 1119-1125.

http://dx.doi.org/10.1016/S0008-6223(00)00314-6

[15] M. Morsy, S. Alsayed and M. Aqel, "Hybrid Effect of Carbon Nanotube and Nano-clay on Physico-Mechanical Properties of Cement Mortar," Construction and Building Materials, Vol. 25, No. 1, 2011, pp. 145-149. http://dx.doi.org/10.1016/j.conbuildmat.2010.06.046

[16] S. Musso, J.-M. Tulliani, G. Ferro and A. Tagliaferro, "Influence of Carbon Nanotubes Structure on the Mechanical Behavior of Cement Composites," Composites Science and Technology, Vol. 69, No. 11-12, 2009, pp. 1985-1990.

http://dx.doi.org/10.1016/j.compscitech.2009.05.002

[17] I. Campillo, A. Guerrero, J. S. Dolado, A. Porro, J. A. Ibáñez and S. Goñi, "Improvement of Initial Mechanical Strength by Nanoalumina in Belite Cements,” Materials Letters, Vol. 61, No. 8, 2007, pp. 1889-1892.

http://dx.doi.org/10.1016/j.matlet.2006.07.150

[18] Z. Li, H. Wang, S. He, Y. Lu and M. Wang, "Investigations on the Preparation and Mechanical Properties of the Nano-Alumina Reinforced Cement Composite,” Materials Letters, Vol. 60, No. 3, 2006, pp. 356-359. http://dx.doi.org/10.1016/j.matlet.2005.08.061

[19] H. Li, M. Zhang and J. Ou, “Abrasion Resistance of Concrete Containing Nano-Particles for Pavement," Wear, Vol. 260, No. 11, 2006, pp. 1262-1266.

http://dx.doi.org/10.1016/j.wear.2005.08.006

[20] H. Li, M. Zhang and J. Ou, "Flexural Fatigue Performance of Concrete Containing Nano-Particles for Pavement," International Journal of Fatigue, Vol. 29, No. 7, 2007, pp. 1292-1301. 
http://dx.doi.org/10.1016/j.ijfatigue.2006.10.004

[21] Y. Qing, Z. Zenan, K. Deyu and C. Rongshen, "Influence of $\mathrm{Nano}_{-} \mathrm{SiO}_{2}$ Addition on Properties of Hardened Cement Paste as Compared with Silica Fume," Construction and Building Materials, Vol. 21, No. 3, 2007, pp. 539545. http://dx.doi.org/10.1016/j.conbuildmat.2005.09.001

[22] J. Vera-Agullo, V. Chozas-Ligero, D. Portillo-Rico, M. García-Casas, A. Gutiérrez-Martínez, J. Mieres-Royo and J. Grávalos-Moreno, "Mortar and Concrete Reinforced with Nanomaterials," Nanotechnology in Construction, Vol. 3, 2009, pp. 383-388.

[23] W. Wei and X. Qu, "Extraordinary Physical Properties of Functionalized Graphene,” Small, Vol. 8, No. 14, 2012, pp. 2138-2151. http://dx.doi.org/10.1002/smll.201200104

[24] K. M. F. Shahil and A. A. Balandin, "Thermal Properties of Graphene and Multilayer Graphene: Applications in Thermal Interface Materials," Solid State Communications, Vol. 152, No. 15, 2012, pp. 1331-1340. http://dx.doi.org/10.1016/j.ssc.2012.04.034

[25] A. V. Eletskii, I. M. Iskandarova, A. A. Knizhnik and D. N. Krasikov, "Graphene: Fabrication Methods and Thermophysical Properties, Physics-Uspekhi, Vol. 54, No. 3 2011, pp. 227-258. http://dx.doi.org/10.3367/UFNe.0181.201103a.0233

[26] P. A. Basnayaka, M. K. Ram, E. K. Stefanakos and A. Kumar, "Supercapacitors Based on Graphene-Polyaniline Derivative Nanocomposite Electrode Materials,” Electrochimica Acta, Vol. 92, 2013, pp. 376-382. http://dx.doi.org/10.1016/j.electacta.2013.01.039
[27] H. Gómez, M. K. Ram, F. Alvi, P. Villalba, E. L. Stefanakos and A. Kumar, "Graphene-Conducting Polymer Nanocomposite as Novel Electrode for Supercapacitors," Journal of Power Sources, Vo. 196, No. 8, 2011, pp. 4102-4108.

http://dx.doi.org/10.1016/j.jpowsour.2010.11.002

[28] F. Alvi, M. K. Ram, P. A. Basnayaka, E. Stefanakos, Y. Goswami and A. Kumar, "Graphene- Polyethylenedioxythiophene Conducting Polymer Nanocomposite Based Supercapacitor,” Electrochimica Acta, Vol. 56, No. 25, 2011, pp. 9406-9412. http://dx.doi.org/10.1016/j.electacta.2011.08.024

[29] "Standard Test Method forMeasurement of Heat of Hydration of Hydraulic Cementitious Materials Using Isothermal ConductionCalorimetry," ASTM C1702-09a, ASTM International, 2012,

[30] P. S. Gaal, M. A. Thermitus and D. E. Stroe, "Thermal Conductivity Measurements using the Flash Method," Journal of Thermal Analysis and Calorimetry, Vol. 78, No. 1, 2004, pp. 185-189. http://dx.doi.org/10.1023/B:JTAN.0000042166.64587.33

[31] "Standard Specification for Portland Cement," ASTM C150, ASTM International, 2012.

[32] W. K. Brown and K. H. Wohletz, "Derivation of the Weibull Distribution Based on Physical Principles and its Connection to the Rosin-Rammler and Lognormal Distributions," Journal of Applied Physics, Vol. 78, No. 4, 1995, pp. 2758-2763. http://dx.doi.org/10.1063/1.360073 